\title{
Magnet Design for the DARHT Linear Induction Accelerators
}

\author{
Michael Burns, Ken Chellis, Cathy Mockler, Tom Tucker, George Velasquez \\ Los Alamos National Laboratory, P.O. Box 1663, Los Alamos, NM 87544 \\ Roger Van Maren \\ Lawrence Livermore National Laboratory, P.O. Box 808, Livermore, CA 94550
}

\section{Abstract}

The linear induction accelerator focus magnet design for the Dual Axis Radiographic Hydrotest (DARHT) facility is presented. Prototype solenoid magnets have demonstrated less than $1 \mathrm{mrad}$ peak transverse error field while using large copper conductors. The design features multi filar winding to reduce field errors due to winding geometry, iron rings within the solenoid to greatly reduce the effects of winding errors, and low profile dipole trim magnets to correct installation crrors. Numerical modeling of several designs is presented as well as data from magnetic field mcasurements of a series of prototype magnets.

\section{INTRODUCTION}

The DARHT facility at Los Alamos will generate intense $\mathrm{x}$-ray pulses for radiography using two Linear Induction Accelerators (LIA). Each LIA will produce a 3-kA, 16 to 20$\mathrm{MeV}, 60$-ns flattop, high-brightness electron beam using a series of $250-\mathrm{kV}$, ferrite-core induction cells. To transport the clectron beam, each cell contains a solenoid magnet with a 2 $\mathrm{kG}$ peak design field. These magnets have been mentioned elsewhere [1] and their design will be discussed in detail here. A view of the final magnet design within the cell is shown in Figure 1.

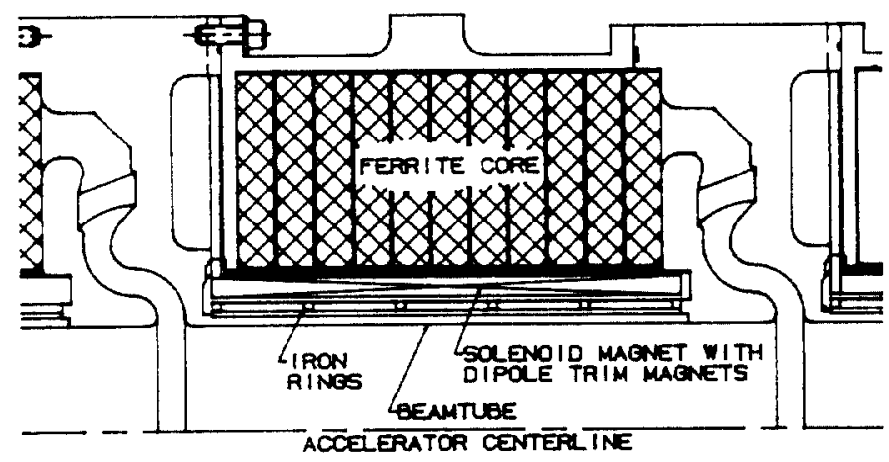

Fig. 1. DARHT accelerator cell and magnet assembly

The reduction of transverse dipole magnetic fields ("error fields") is the principal design challenge and the focus of this paper. Error fields will deflect the electron beam from the accelerator centerline, causing the chromatic aberration known as "corkscrew" [2] and enhancing the growth of the Beam Brcakup instability (BBU). Both phenomena result in transverse oscillations of the electron beam, which increases the time-integrated beam diameter at the final focus and therefore the radiographic spot size.

Section 2 of this paper discusses the use of multiple conductor winding patterns to reduce error fields inherent in the winding geometry. Section 3 describes iron homogenizer rings placed within the solenoid to reduce the error fields caused by winding asymmetries. Magnetic field measurements of a series of prototype magnets are presented in section 4 .

\section{WINDING GEOMETRY}

Transverse error fields may be caused by the use of a winding pattem in which the magnet leads and conductor crossovers produce dipole fields on the solenoid axis. The conventional monofilar (one conductor per layer), layer-wound solenoid is such a winding pattern. W.E. Nexsen [3] has shown that the use of several conductors per layer arranged so that the conductors have at least two-fold symmetry relative to the solenoid axis results in a winding geometry without error fields on axis. We refer to such multi conductor winding geometries as multi filar.

A bifilar (two conductors per layer) coil that reduces winding pattern error fields is realized by placing the two conductors $180^{\circ}$ apart from each other during the winding process. A quadrufilar (four conductors per layer) geomerry maintains a separation of 90 degrees between conductors.

We have investigated monofilar, bifilar, and quadrufilar geometries with the three-dimensional magnetic field code MAFCO. This code uses the Biot-Savart law to calculate the magnetic field at a point in space by adding the contributions of every element of the coil.

Figure 2 is a plot of the magnetic-axis tilt along the centerline of a monofilar and bifilar coil sized for DARHT. The magnetic-axis tilt is defined as the magnitude of the dipole field on axis divided by the magnitude of the solenoidal field at that point. To meet the DARHT performance goals, we require that random magnetic axis tilts be less than $2 \mathrm{mrad}$ along the accelerator. To allow tolerance for the alignment system, we desire coils with random errors less than $1 \mathrm{mrad}$. As Fig. 2 shows, there are appreciable errors in the monofilar geometry. The configuration of the leads and cross overs creates a dipole field on axis. The error fields due to winding geometry have been reduced in the bifilar design however. Therefore, a multi-filar winding pattern was selected for DARHT. 


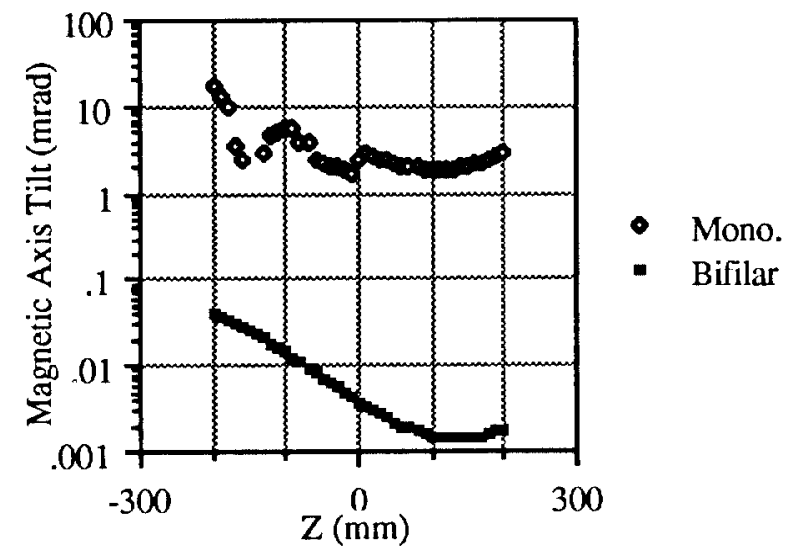

Fig. 2. Calculated magnetic axis tilt for two winding patterns. The coil leads for these DARHT-sized solenoids are near the $-160 \mathrm{~mm}$ position.

\section{HOMOGENIZER RINGS}

Winding errors, or symmetry-breaking random conductor location errors, reduce the effectiveness of the multi filar winding geometry and can cause significant error fields. Following Nexen [3], we estimate that a local 1-mrad error can be caused in a DARHT solenoid by a conductor location error of $0.17 \mathrm{~mm}$ in the first laycr. Such locational accuracy is not readily achieved with conventional winding techniques.

It is common for high precision solenoids to be constructed with a great number of turns so that random location errors tend to cancel. This requires comparatively small wire, and the resulting high resistance often dictates the use of superconducting technology. High reliability and low cost are fundamental requirements for the DARHT magnets, and we therefore wish to use conventional, square cross-section hollow conductor. This results in a relatively small number of turns and increased sensitivity to winding errors compared to small wire.

Feinberg, et. al. [4] have shown that placing a scries of annular iron rings within the solenoid windings reduces error ficlds in the region inside the rings. Each ring forms a magnetic equipotential surface that eliminates the azimuthal asymmetries causing the error ficlds. The rings are spaced far enough apart along the coil axis that they do not interfere with the solenoidal field. For the DARHT magnets, $6.35 \mathrm{~mm}$ square cross-section rings axially spaced $63.5 \mathrm{~mm}$ apart were considered. These small rings are shown in Fig. 1.

The effect of the iron homogenizer rings was studied with the three-dimensional, magnetic field code MAGNUS. Figure 3 shows the calculated magnetic axis tilt for an ideal solenoid tilted $17.5 \mathrm{mrad}$ (one degrec) relative to the axis of six coaxial iron rings. The rings reduce the magnetic axis tilt by a factor of 5.5. Roughly the same reduction factor was calculated for tilts of 8.7 and $34.9 \mathrm{mrad}$.

The effect of the homogenizers on the solenoidal field was studied with the two-dimensional magnetic ficld code POISSON. At the beamtube wall the axial field varied roughly $2 \%$, increasing between rings and decreasing near the rings. The radial profile of the solenoidal field was the same shape with and without rings, although the magnitude of the axial field varied slightly near the rings.

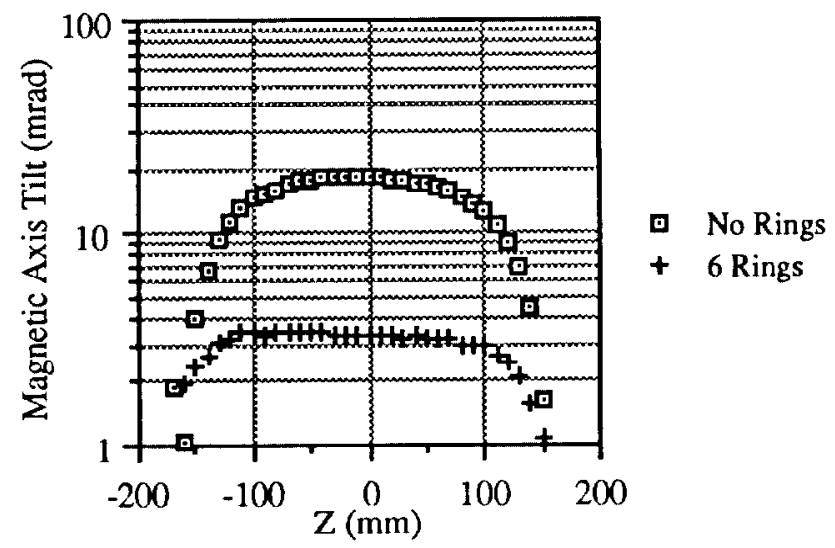

Fig. 3. Calculated magnetic axis tilt of an ideal solenoid with and without homogenizer rings for a $1^{\circ}$ coil tilt with respect to the ring axis.

Inclusion of the homogenizer rings within the DARHT solenoid requires the removal of two layers of the magnet because the inner and outer radii of the solenoid are fixed by the cell design (Fig. 1). Thus, the power dissipated by the coil increases by a factor of two. A quadrufilar coil provides sufficient cooling, however, for $2-k G$ operation. The quadrufilar geometry increases the number of parallel water circuits by a factor of four compared to the monofilar geometry, and the length of each is reduced by a factor of four.

The magnetic axis of the solenoid is determined predominantly by the axes of the homogenizer rings. Accurate placement of the rings within the accelerator cell assures colinearity of the magnetic and mechanical axes. Magnetic alignment is thus reduced to a more straightforward mechanical alignment effort.

\section{MAGNETIC FIELD MEASUREMENTS}

To measure the effect of winding geometries, four DARHT-type solenoids were constructed. In addition to a monofilar, a bifilar, and a quadrufilar coil, we fabricated a monofilar solenoid in which the outer two layers were rotated $180^{\circ}$ relative to the inner layers. This provided for partial cancellation of the error fields associated with the leads and is referred to as the "nested" coil.

Each coil is layer-wound from four layers of $6.73 \mathrm{~mm}$. square-hollow copper conductor. The coils were wound on a $6.35 \mathrm{~mm}$. wall stainless steel tube with a $148.2 \mathrm{~mm}$. bore. The length of each coil was $323.9 \mathrm{~mm}$.

Cosine-wound dipole trim magnets were included with each solenoid. These are similar to magnets used in the Los Alamos Ground Test Accelerator program [5] and were fabricated by photoetching $0.53 \mathrm{~mm}$ thick copper sheet laminated to $0.13 \mathrm{~mm}$. kapton insulation. Each dipole had two such layers and a radial thickness of approx, $1.5 \mathrm{~mm}$. The 
dipoles were wrapped around the solenoid magnet and oriented $90^{\circ}$ apart. Therefore, the trim magnets could produce a dipole field of arbitrary phase angle to correct for solenoid installation crrors.

The magnetic axis tilt was measured with the apparatus described in Ref. 3. A Hall probe, oriented to measure radial ficlds, was rotated on the axis of the solenoid. The Hall probe signal was recorded every $5^{\circ}$ of rotation to determine the magnitude and phase of the dipole error field at a point along the solenoid axis. To determine the magnetic axis tilt, this measurement was compared to the solenoidal field measured at that point with a separate Hall probe. The process was repeated at several points along the coil axis.

This measurement determined the magnetic axis tilt with respect to the Hall probe rotation axis. The alignment of the measurement equipment with the bore of the solenoid is sufficiently good that the measurement resolution is of the order of a few tenths of a mrad..

Figure 4 is a plot of the magnetic axis tilt along the centerline of the four magnets not having homogenizer rings. As expected, the monofilar geometry had the largest error. The measurement of this coil compares favorably with the calculation shown in Fig 2. The rotation of the outer two layers in the nested coil resulted in a significant improvement. The bifilar and quadrufilar crrors are similar and are probably dominated by winding errors. Even so, the multi filar geometries provide about a factor of three improvement over to the monofilar coil.

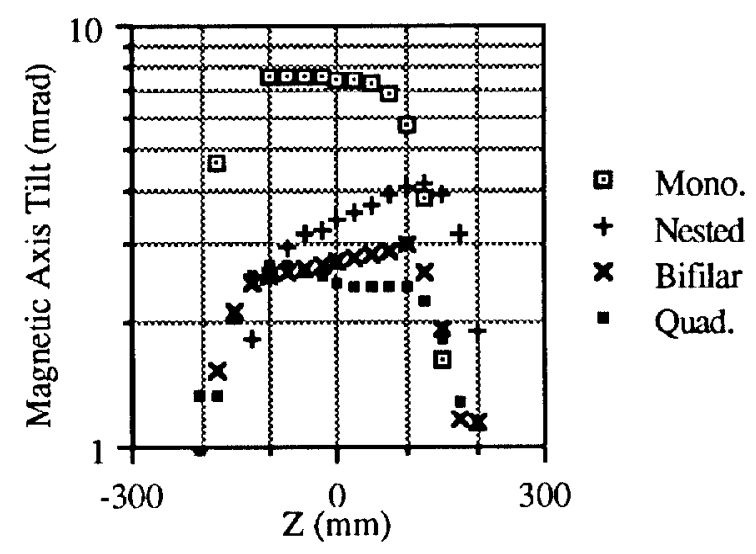

Fig. 4. Measured magnetic axis tilt for solenoids with different winding patterns.

We next measured three solenoids, each with six iron homogenizer rings. For each magnet an aluminum winding mandrel was fabricated with a carefully machined inside diameter. A set of six, match-ground iron (C1008) rings were then shrunk in liquid nitrogen, placed within the mandrel, and allowed to expand into an interference fit. The alignment of the rings with respect to the mounting surfaces of the mandrel was excellent. Typically, the rings and mounting surfaces were coaxial to within less than $0.013 \mathrm{~mm}$. A two-layer, quadrufilar solenoid was then wound on the mandrel using the same conductor as before. Finally, the coil/ring assembly was slid over a stainless stecl bore tube on which were wrapped the dipole trim magnets.

Figure 5 shows the results of the measurements of the scries of threc solenoids with iron homogenizer rings. A complete measurement through a solenoid required two separate mechanical set ups and the repeatability of each was no better than $0.2 \mathrm{mrad}$. This can be seen as the "bump" in each curve near the middle of the magnet. The overall spread between magnets may be due to different sets of winding errors and the accuracy of the measurement equipment. The results indicate that the magnetic axis tilt near the center of the solenoids was 0.5 to $0.6 \mathrm{mrad}$, which is better than an order of magnitude better than the conventional monofilar solenoid.

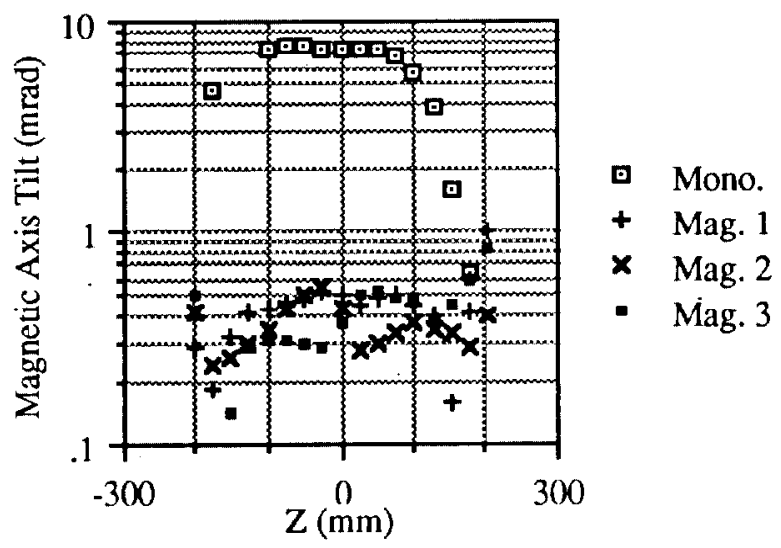

Fig. 5. Magnetic axis tilt of a series of solenoids with iron homogenizer rings. A conventional, monofilar solenoid is also shown for comparison.

The dipole trim magnets are located inside the iron rings so that their fields are not affected by the homogenizers. The smaller radii and the presence of the rings increases the field generated by the trim magnets. A single trim magnet within the homogenizers produced an integrated dipole field of 0.58 $\mathrm{kG}-\mathrm{cm}$. A trim magnet wrapped around the 4-layer solenoids without iron rings generated an integrated dipole field of 0.40 kG-cm.

\section{REFERENCES}

[1] G.J. Caporaso and A.G. Cole, "Design of Long Induction Linacs", 1990 Linear Accelerator Conference, Albuquerque, NM, September 1990.

[2] Y.J. Chen, "Corkscrew Modes in Linear Accelerators", Nucl. Inst. and Meth. in Phys. Res. A, vol. 292, pp455, 1990.

[3] W.E. Nexsen, "Characterization of Field Errors of Layer Wound Short Solenoids", 1990 Linear Accelerator Conference, Albuquerque, NM, September 1990.

[4] B. Feinberg, I.G. Brown, K. Halbach, and W.B. Kunkel, "A Method for Improving the Quality of the Magnetic Field in a Solenoid", Nuc. Inst. and Meth., vol. 203, pp 81-85, 1982.

[5] G. Lawrence, A. Jason, and B. Blind, "Dipole, Quadrupole, Scxtupolc, and Octupole Magnetic Fields from Cosine-Wound Printed Circuit Coils", Los Alamos National Laboratory AT-3 Technical Note 86-128. 